\title{
Role of Radiotherapy in Modern Treatment of Hodgkin's Lymphoma
}

\author{
Kheng-Wei Yeoh and N. George Mikhaeel \\ Department of Clinical Oncology, Guy's and St. Thomas' Hospital, London, UK \\ Correspondence should be addressed to Kheng-Wei Yeoh, kwyeoh@doctors.org.uk \\ Received 21 July 2010; Accepted 16 September 2010 \\ Academic Editor: Nelson J. Chao
}

Copyright ( $) 2011$ K.-W. Yeoh and N. G. Mikhaeel. This is an open access article distributed under the Creative Commons Attribution License, which permits unrestricted use, distribution, and reproduction in any medium, provided the original work is properly cited.

\begin{abstract}
Hodgkin's Lymphoma was incurable until the advent of effective therapeutic radiation around the first half of the 20th century. As survival rates improved, the long-term toxicities from radiotherapy began to emerge. This together with the availability of effective chemotherapy has encouraged a combined modality approach for early-staged disease and the omission of radiotherapy in advanced-staged disease. The differing toxicities of radiotherapy and chemotherapy has promoted ongoing research to identify the utility of each of these modalities in the modern management of Hodgkin's Lymphoma. This article will provide a critical review of the developments and indications for modern radiotherapy, in context with advances in chemotherapy, for the treatment of Hodgkin's Lymphoma.
\end{abstract}

\section{Introduction}

During the first half of the 20th century, Hodgkin's Lymphoma (HL) was incurable until the development of effective therapeutic radiation [1]. It involved irradiating beyond the site of disease to include the adjacent nodal groups, the so-called extended field radiotherapy (EFRT) with total or subtotal nodal irradiation [2]. With Radiotherapy (RT) alone, 10 -year relapse-free survival of around $70 \%$ had been achieved in early-staged HL $[3,4]$.

However, advanced stage HL remained less curable with RT even with subtotal or total nodal RT until the introduction of combination chemotherapy. With the advent of effective chemotherapy regimes in the 1980s with MOPP [5] and ABVD [6], better cure rates were achieved in advanced disease establishing chemotherapy as the main line of treatment in advanced disease. Gradually, chemotherapy use moved to earlier-stage disease, initially as a pre-RT treatment in bulky mediastinal cases to reduce the extent of mediastinal RT and later to improve the outcome of RT alone. With emerging reports of long-term toxicities from EFRT, namely with pulmonary, cardiac toxicity, and secondary malignancies [7-13] together with studies showing that limited RT was sufficient after prior chemotherapy, combined modality treatment has been established in early disease. More recently, there has been several opinions calling on treating early stage HL with chemotherapy alone without $\mathrm{RT}$, and this remains an interesting research question.

The paper below will aim to highlight the developments of RT in HL treatment, with specific focus on its role in early, advanced, and relapsed disease.

\section{Developments in RT}

2.1. Background. The concept of RT to cure HL by Gilbert [14] was implemented with kilovoltage [15] and later with megavoltage machines [1]. Doses of up to $4500 \mathrm{rads}$ (1000 rads/wk) were commonly used to treat extended fields in order to achieve total lymphoid irradiation (TLI). This included mantle field, inverted-Y techniques, and modifications to include splenic irradiation, delivered with a parallel opposed technique. It was also not uncommon to irradiate liver and lungs involved with disease; all this was done when staging laparotomy and splenectomy were standard practice.

In the light of RT toxicities, suboptimal relapse and survival rates together with the introduction of effective chemotherapy, the impetus to rationalise RT delivery became 
a priority. This was achieved through prognostic risk stratification, combined modality approach with chemotherapy in concert with advances in RT. These include improvements with RT techniques and rationalisation in both the irradiated volume as well as the RT doses used.

Risk stratification was introduced in the 1980s originally by the BNLI group [16], and also the EORTC and GHSG groups to rationalise treatment for good prognostic-HL patients in order to minimise toxicity. The EORTC/GELA and GHSG groups have identified several features indicative of unfavourable prognosis $[17,18]$ (Table 1 ) in early stage HL which has allowed studies to investigate risk stratified treatment.

Advanced disease is classified as stage 3 and 4 disease and also includes stage $2 \mathrm{~B}$ disease in presence of bulky mediastinal or extranodal disease in the GHSG classification.

2.2. RT Doses. From the traditional doses of over 40$45 \mathrm{~Gy}$ used, doses for treatment of HL have been rationally reduced in order to maintain optimal outcome with reduced toxicities. Doses of $30 \mathrm{~Gy}$ have been shown to yield similar results to higher doses used with no increased dose-response relationship seen for higher doses used [19-21], and as such $30 \mathrm{~Gy}$ in combined modality treatment (CMT) has become the standard in most countries. Lower doses have also been shown to be effective from 2 current studies, one by the EORTC/GELA group and the other the GHSG group. The ongoing EORTC/GELA H9F trial had 20 Gy Involved Field RT (IFRT) in one trial arm which showed similar results to higher doses of radiotherapy in combined modality treatment of early-staged HL [22]. Incidentally, the arm where RT was omitted has been stopped due to higher relapse rates found. Preliminary results from the ongoing GHSG HD10 trial has shown that dose reduction to 20 Gy IFRT as part of combined modality treatment showed equivalent results to higher doses [23].

2.3. RT Volume. As well as the ongoing reduction in RT doses being used, smaller RT fields have been used and several randomised trials have shown that IFRT, where only the nodal region is irradiated, is as effective as EFRT with equivalent overall survival rates [24-27]. Further progress has gone beyond the concept of IFRT to Involved Site RT (ISRT) and now Involved Node RT (INRT), where only the involved nodes are irradiated, have been proposed by the EORTC/GELA group [28]. Encouraging results have come from a retrospective series showing equivalent progressionfree survival (PFS) and overall survival (OS) for INRT compared to IFRT [29].

2.4. RT Technology. In concert with the above developments, there has been significant improvement in RT techniques in order to achieve more conformal radiotherapy treatment from the traditional large parallel opposed fields used. This has allowed RT to progress to $2 \mathrm{D}$ conformal [30] and to now commonly used 3D conformal techniques [31], with Intensity-modulated RT (IMRT) being shown to be able to further improve target volume conformity with reductions of dose to critical structures [32]. These have therefore allowed the developments of reduction in irradiated volume as described above.

\section{Role of RT in Early Disease}

The improvement of clinical staging with the introduction of the Cotswolds modifications of the Ann Arbour staging in 1989 [33] together with adverse outcomes being reported with the routine use of staging laparotomy and splenectomy with no clinical benefit [16] led to recommendations to abandon these procedures. Clinical risk stratification identifying favourable and unfavourable groups within earlystaged HL by groups such as the EORTC and GHSG has allowed investigation to rationalise treatment in early $\mathrm{HL}$ further.

Results from several trials support CMT for the treatment of early-staged HL.

Studies comparing CMT with RT alone have shown superiority of CMT. These have been shown by both the GHSG and EORTC groups. In the EORTC H8F trial, patients with early-staged favourable HL were randomised to either 3 cycles of MOPP/ABV chemotherapy followed by IFRT of 36 Gy or to RT alone with STNI. The results showed a better event-free survival (EFS) and OS in favour of the CMT arm (EFS $98 \%$ versus $74 \%, 10$ yr OS $97 \%$ versus $92 \%$ ) [26]. These results are supported by the GHSG HD7 trial favouring 2 cycles of ABVD chemotherapy followed by RT versus RT alone in early-staged favourable HL [34].

Studies comparing CMT with chemotherapy alone have shown that chemotherapy alone yields worse outcomes. The EORTC/GELA H9F trial compared 6 cycles of chemotherapy with or without IFRT [22]. The arm without RT was closed early due to higher-than-expected relapse rates. Similar findings were reported in the CCG 5942 trial comparing 4-6 cycles of chemotherapy with or without RT. The arm without RT was closed early due to a significantly higher number of relapses seen on the no-radiotherapy arm [35]. Two North American studies comparing chemotherapy alone versus CMT showed an inferior PFS and freedom from progression (FFP) in the chemotherapy alone arms with the NCIC/ECOG HD-6 Trial showing a 5-Year PFS of 87\% versus $93 \%$, in favour of CMT [36] and the MSKCC trial showing a FFP of $86 \%$ versus $81 \%$ [37] in favour of CMT. Advantages in overall survival for CMT were shown in the Tata Memorial Trial which showed an 8 year EFS of $88 \%$ versus $76 \%$ and OS of $100 \%$ versus $89 \%$ in favour of CMT versus 6 cycles of ABVD alone [38]. Although all stages of HL were included in this trial, $55 \%$ of enrolled patients had early HL.

Based on these results and data supporting ABVD over other combination chemotherapy regimes [39-42], the recommendations for the treatment of early HL are with the combined modality approach with 2 to 4 cycles of ABVD followed by IFRT of 30 Gy $[43,44]$ with the ESMO group making further recommendations that favourable early HL patients should receive 2 cycles of ABVD in combination with IFRT whilst 4 cycles of ABVD in combination with IFRT being reserved for the unfavourable group. Further dose deescalation studies for favourable early HL are underway such 
TABLE 1: EORTC/GELA and GHSG risk factors for early-staged HL.

\begin{tabular}{ll}
\hline Early-staged (I-II) unfavourable features (EORTC/GELA) & Early-staged (I-II) unfavourable features (GHSG) \\
\hline Four or more nodal areas involved & Three or more nodal areas involved \\
Bulky mediastinum* & Bulky mediastinum* \\
ESR $>50$ without B symptoms or $>30$ with B symptoms & ESR $>50$ without B symptoms or $>30$ with B symptoms \\
Aged 50 years old or more & Extranodal disease \\
\hline
\end{tabular}

*Bulky mediastinum defined as mediastinal/thoracic ratio $>0.35$.

as the NCRN RAPID trial in the UK using information from interval PET imaging. The prognostic information from interval PET scanning in HL [45] has also formed the basis for the GHSG HD16 trial and EORTC 10F trial. The use of PET imaging is also now becoming standard for routine staging of HL [46] as well as gaining support for use in RT planning with INRT [47].

Some controversy has arisen over the proposition for the use of chemotherapy alone in the management of early HL [48-50] lending support for this approach to be within an option of the NCCN guidelines for the management of early HL [51]. The impetus for this approach was based on toxicity data during the era where EFRT was routine. There remains no evidence to support this approach with worse reported outcomes when RT is omitted, as highlighted from the various studies discussed earlier. A recent meta-analysis studying 5 randomised control trials [52] has confirmed the superiority of CMT over chemotherapy alone with a hazard ratio (HR) of 0.41 for tumour control and 0.40 for OS for patients receiving CMT compared to chemotherapy alone. As such the standard of care for early HL remains CMT.

A caveat for radical treatment with radiotherapy alone is with Nodular Lymphocyte Predominant Hodgkin's Lymphoma (NLPHL) where 30 Gy has been shown to yield similar results compared with combined-modality treatment for Stage 1A NLPHL with no risk factors whilst advanced NLPHL has been treated with classic HL protocols $[53,54]$.

\section{Role of RT in Advanced Disease}

In the prechemotherapy era, advanced HL was treated with Total Lymph Node Irradiation (TLI) where the majority of patients relapsed and died. With the advent of MOPP, long term remission of around 50\% were achieved [55]. This was then replaced by ABVD after randomised trials showed superiority over other regimes including MOPP $[56,57]$. Several other regimes have also been shown to be more toxic but no more effective than ABVD [39-42, 58 ] with 3-year OS survival rates of around 90\% being achieved. Based on these findings, ABVD has now become the standard arm for comparison in ongoing trials. But with suboptimal long-term failure-free and survival rates, dose escalated regimes have been used to improve results [59], however not without increased toxicities. Direct comparison of escalated BEACOPP with the standard ABVD regime is ongoing with the EORTC 20012 trial.

The Stanford V protocol offered an alternative regime with a short period of 12 weeks of chemotherapy followed by
RT to bulky sites, classified as $5 \mathrm{~cm}$ or more [60]. However, comparison of the Stanford $\mathrm{V}$ regime with ABVD and MOPPEBVCAD showed worse PFS [61]. Results are awaited for the UK NCRI Stanford V versus ABVD trial for advanced HL.

Offering RT after effective chemotherapy is not standard practice and is still undergoing investigation. However, data so far can allow risk stratification into groups whom may benefit. Although a meta-analysis [62] and studies by the GELA [63] and EORTC groups [64] showed no benefit of consolidation RT after effective chemotherapy with suggestions of worsened outcome when RT was added, more recent data have emerged from 2 large randomised control trials (RCT) in support of consolidation RT. The UKLG LY09 trial showed 5 year PFS of $71 \%$ versus $86 \%$, with OS HR of 0.47 in favour of consolidation RT [65]. This concurs with another large RCT from Tata Memorial Hospital showing EFS and OS advantage for consolidation RT after 6 cycles of ABVD chemotherapy in all stages of HL [38]. Furthermore, most of the trials of chemotherapy dose escalation include RT for most patients in their protocols. Utilising the extra data obtained from PET to identify metabolically active residual masses postchemotherapy, the GHSG HD15 trial incorporates PET to assess residual lesions $>2.5 \mathrm{~cm}$ to receive consolidation RT, and preliminary results have been able to show favourable negative predictive value of PET of $94 \%$ [66] hence offering the potential towards a risk stratified approach.

\section{Role of RT in Salvage Treatment}

Although the standard treatment for patients with refractory and relapsed HL to chemotherapy and combined chemoradiotherapy is high-dose therapy supported by autologous stem-cell transplant, RT has a role in the peritransplantation setting. Results by Poen et al. at Stanford [67] has shown that most relapses occurred in areas of known disease pretransplant and posttransplant irradiation resulted in no in-field relapses. This concept has been supported by a series at MSK showing favourable results [68, 69]. Results from clinical trials in this setting as well as studies looking at the utility of PET to identify peritransplant active disease should yield exciting recommendations.

\section{Conclusion}

From the above review, it can be seen that the role of RT in the management of HL has changed considerably 
over the last century with the advent of better staging, risk stratification strategies, effective chemotherapy and salvage regimens, and the progress in RT techniques and technology. RT doses and volume have been significantly rationalised to minimise toxicities with the establishment of highly conformal RT techniques whilst allowing concepts such as INRT to be introduced.

It is important to highlight that studies which have shown toxicities with RT have been based on historical series where obsolete nonconformal RT with higher doses were used and that survival data reported in trials may be misrepresented with the prevalence and success rates of salvage chemotherapy regimes and transplantation in relapsed $\mathrm{HL}$.

In early-staged HL, CMT is thus the established standard of care with adjustments in the number of chemotherapy cycles justified according to the risk factors. In advancedstaged HL, a full course of chemotherapy is the standard with special circumstances advocating consolidation RT, namely initial bulk disease or residual PET avidity. Ongoing trials will no doubt clarify the role of RT in these situations as well as identifying the role of PET to dictate consolidation RT.

Ongoing work with the above strategies of achieving long-term cure whilst minimising toxicities, whether it be from radiotherapy or chemotherapy, should continue to yield promising results.

\section{References}

[1] H. S. Kaplan, "The radical radiotherapy of regionally localized Hodgkin's disease," Radiology, vol. 78, pp. 553-561, 1962.

[2] H. S. Kaplan, "Prognostic significance of the relapse-free interval after radiotherapy in Hodgkin's disease," Cancer, vol. 22, no. 6, pp. 1131-1136, 1968.

[3] R. T. Hoppe, C. N. Coleman, and R. S. Cox, "The management of stage I-II Hodgkin's disease with irradiation alone or combined modality therapy: the Stanford experience," Blood, vol. 59, no. 3, pp. 455-465, 1982.

[4] P. Mauch, N. Tarbell, H. Weinstein et al., "Stage IA and IIA supradiaphragmatic Hodgkin's disease: prognostic factors in surgically staged patients treated with mantle and paraaortic irradiation," Journal of Clinical Oncology, vol. 6, no. 10, pp. 1576-1583, 1988.

[5] V. T. Devita Jr., A. A. Serpick, and P. P. Carbone, "Combination chemotherapy in the treatment of advanced Hodgkin's disease," Annals of Internal Medicine, vol. 73, no. 6, pp. 881895, 1970.

[6] G. Bonadonna, R. Zucali, and S. Monfardini, "Combination chemotherapy of Hodgkin's disease with adriamycin, bleomycin, vinblastine, and imidazole carboxamide versus MOPP," Cancer, vol. 36, no. 1, pp. 252-259, 1975.

[7] B. M. P. Aleman, A. W. Van Den Belt-Dusebout, W. J. Klokman, M. B. Van't Veer, H. Bartelink, and F. E. Van Leeuwen, "Long-term cause-specific mortality of patients treated for Hodgkin's disease," Journal of Clinical Oncology, vol. 21, no. 18, pp. 3431-3439, 2003.

[8] J. M. Cosset, M. Henry-Amar, J. H. Meerwaldt et al., "Longterm toxicity of early stages of Hodgkin's disease therapy: the EORTC experience-EORTC Lymphoma Cooperative Group," Annals of Oncology, vol. 2, 2, pp. 77-82, 1991.
[9] M. Henry-Amar, M. Hayat, J. H. Meerwaldt et al., "Causes of death after therapy for early stage Hodgkin's disease entered on EORTC protocols," International Journal of Radiation Oncology Biology Physics, vol. 19, no. 5, pp. 1155-1157, 1990.

[10] A. K. Ng, M. P. Bernardo, E. Weller et al., "Long-term survival and competing causes of death in patients with early-stage Hodgkin's disease treated at age 50 or younger," Journal of Clinical Oncology, vol. 20, no. 8, pp. 2101-2108, 2002.

[11] F. E. Van Leeuwen, W. J. Klokman, M. B. Van't Veer et al., "Long-term risk of second malignancy in survivors of Hodgkin's disease treated during adolescence or young adulthood," Journal of Clinical Oncology, vol. 18, no. 3, pp. 487-497, 2000.

[12] M. Sieber, A. Engert, and V. Diehl, “Treatment of Hodgkin's disease: results and current concepts of the German Hodgkin's Lymphoma Study Group," Annals of Oncology, vol. 11, no. 1, pp. S81-S85, 2000.

[13] M. M. O’Brien, S. S. Donaldson, R. R. Balise et al., "Second malignant neoplasms in survivors of pediatric Hodgkin's lymphoma treated with low-dose radiation and chemotherapy," Journal of Clinical Oncology, vol. 28, no. 7, pp. 1232-1239, 2010.

[14] R. Gilbert, "Radiotherapy in Hodgkin's disease. (Malignant Granulomatosis); anatomic and clinical foundations; governing principles; results," American Journal of Roentgenology, vol. 41, p. 198, 1939.

[15] M. V. Peters, "A study of survivals in Hodgkin's disease treated radiologically," American Journal of Roentgenology, vol. 63, p. 299, 1950.

[16] J. L. Haybittle, F. G. J. Hayhoe, and M. J. Easterling, "Review of British National Lymphoma Investigation studies of Hodgkin's disease and development of prognostic index," Lancet, vol. 1, no. 8435, pp. 967-972, 1985.

[17] M. Tubiana, M. Henry-Amar, P. Carde et al., "Toward comprehensive management tailored to prognostic factors of patients with clinical stages I and II in Hodgkin's disease. The EORTC Lymphoma Group controlled clinical trials: 19641987," Blood, vol. 73, no. 1, pp. 47-56, 1989.

[18] V. Diehl, M. Pfreundschuh, M. Loffler et al., "Chemotherapy of Hodgkin's lymphoma with alternating cycles of COPP (cyclophosphamide, vincristin, procarbazine, prednisone) and ABVD (doxorubicin, bleomycin, vinblastine and dacarbazine). Reults of the HD1 and HD3 trials of the German Hodgkin Study Group," Medical Oncology and Tumor Pharmacotherapy, vol. 6, no. 2, pp. 155-162, 1989.

[19] K. L. Schewe, J. Reavis, L. E. Kun, and J. D. Cox, "Total dose, fraction size, and tumor volume in the local control of Hodgkin's disease," International Journal of Radiation Oncology Biology Physics, vol. 15, no. 1, pp. 25-28, 1988.

[20] G. E. Hanks, J. J. Kinzie, and R. L. White, "Patterns of care outcome studies. Results of the national practice in Hodgkin's disease," Cancer, vol. 51, no. 4, pp. 569-573, 1983.

[21] T. L. Thar, R. R. Million, R. J. Hausner, and M. H. B. McKetty, "Hodgkin's disease, stages I and II. Relationship of recurrence to size of disease, radiation dose, and number of sites involved," Cancer, vol. 43, no. 3, pp. 1101-1105, 1979.

[22] H. Eghbali, P. Brice, G.-Y. Creemers et al., "Comparison of three radiation dose levels after EBVP regimen in favourable supradiaphragmatic clinical stages (CS) I-II Hodgkin's lymphoma (HL): preliminary results of the EORTC-GELA H9-F trial," Blood, vol. 106, p. 814a, 2005.

[23] A. Pluetschow, A. Engert, H. T. Eich et al., "Combined modality treatment of two or four cycles of ABVD followed by 
involved field radiotherapy in the treatment of patients with early stage Hodgkin's lymphoma: update interim analysis of the randomised HD10 study of the German Hodgkin's Study Group (GHSG)," Blood, vol. 106, p. 2673 a, 2005.

[24] G. Bonadonna, V. Bonfante, S. Viviani, A. Di Russo, F. Villani, and P. Valagussa, "ABVD plus subtotal nodal versus involvedfield radiotherapy in early-stage Hodgkin's disease: long-term results," Journal of Clinical Oncology, vol. 22, no. 14, pp. 2835 2841, 2004.

[25] A Engert, P. Schiller, A. Josting et al., "Involved-field radiotherapy is equally effective and less toxic compared with extendedfield radiotherapy after four cycles of chemotherapy in patients with early-stage unfavourable Hodgkin's lymphoma: results of the HD8 trial of the German Hodgkin's Lymphoma Study Group," Journal of Clinical Oncology, vol. 21, pp. 3601$3608,2003$.

[26] C. Fermé, H. Eghbali, H. Jacobus et al., "Chemotherapy plus involved-field radiation in early-stage Hodgkin's disease," New England Journal of Medicine, vol. 357, no. 19, pp. 1916-1927, 2007.

[27] E. M. Noordijk, P. Carde, N. Dupouy et al., "Combinedmodality therapy for clinical stage I or II Hodgkin's lymphoma: long-term results of the European organisation for research and treatment of cancer $\mathrm{H} 7$ randomized controlled trials," Journal of Clinical Oncology, vol. 24, no. 19, pp. 31283135, 2006.

[28] T. Girinsky, R. van der Maazen, L. Specht et al., "Involvednode radiotherapy (INRT) in patients with early Hodgkin lymphoma: concepts and guidelines," Radiotherapy and Oncology, vol. 79, no. 3, pp. 270-277, 2006.

[29] B. A. Campbell, N. Voss, T. Pickles et al., "Involved-nodal radiation therapy as a component of combination therapy for limited-stage Hodgkin's lymphoma: a question of field size," Journal of Clinical Oncology, vol. 26, no. 32, pp. 5170-5174, 2008.

[30] J. Yahalom and P. Mauch, "The involved field is back: issues in delineating the radiation field in Hodgkin's disease," Annals of Oncology, vol. 13, supplement 1, pp. 79-83, 2002.

[31] P. Diez, P. J. Hoskin, and E. G. A. Aird, "Treatment planning and delivery of involved field radiotherapy in advanced Hodgkin's disease: results from a questionnaire-based audit for the UK Stanford V regimen vs ABVD clinical trial quality assurance programme (ISRCTN 64141244)," British Journal of Radiology, vol. 80, no. 958, pp. 816-821, 2007.

[32] L. Cella, R. Liuzzi, M. Magliulo et al., "Radiotherapy of large target volumes in Hodgkin's Lymphoma: a normal tissue sparing capability of forwards IMRT versus conventional techniques," Radiation Oncology, vol. 5, p. 33, 2010.

[33] T. A. Lister, D. Crowther, S. B. Sutcliffe et al., "Report of a committee convened to discuss the evaluation and staging of patients with Hodgkin's disease: cotswolds meeting," Journal of Clinical Oncology, vol. 7, no. 11, pp. 1630-1636, 1989.

[34] A. Engert, J. Franklin, H. T. Eich et al., "Two cycles of doxorubicin, bleomycin, vinblastine, and dacarbazine plus extended-field radiotherapy is superior to radiotherapy alone in early favorable Hodgkin's lymphoma: final results of the GHSG HD7 trial," Journal of Clinical Oncology, vol. 25, no. 23, pp. 3495-3502, 2007.

[35] J. B. Nachman, R. Sposto, P. Herzog et al., "Randomized comparison of low-dose involved-field radiotherapy and no radiotherapy for children with Hodgkin's disease who achieve a complete response to chemotherapy," Journal of Clinical Oncology, vol. 20, no. 18, pp. 3765-3771, 2002.
[36] R. M. Meyer, M. K. Gospodarowicz, J. M. Connors et al., "Randomized comparison of ABVD chemotherapy with a strategy that includes radiation therapy in patients with limited-stage Hodgkin's lymphoma: national Cancer Institute of Canada Clinical Trials Group and the Eastern Cooperative Oncology Group," Journal of Clinical Oncology, vol. 23, no. 21, pp. 4634-4642, 2005.

[37] D. J. Straus, C. S. Portlock, J. Qin et al., "Results of a prospective randomized clinical trial of doxorubicin, bleomycin, vinblastine, and dacarbazine (ABVD) followed by radiation therapy (RT) versus ABVD alone for stages I, II, and IIIA nonbulky Hodgkin disease," Blood, vol. 104, no. 12, pp. 34833489, 2004.

[38] S. Laskar, T. Gupta, S. Vimal et al., "Consolidation radiation after remission in Hodgkin's disease following six cycles of doxorubicin, bleomycin, vinblastine, and dacarbazine chemotherapy: is there a need?" Journal of Clinical Oncology, vol. 22, no. 1, pp. 62-68, 2004.

[39] G. P. Canellos, J. R. Anderson, K. J. Propert et al., "Chemotherapy of advanced Hodgkin's disease with MOPP, ABVD, or MOPP alternating with ABVD," New England Journal of Medicine, vol. 327, no. 21, pp. 1478-1484, 1992.

[40] S. Viviani, G. Bonadonna, A. Santoro et al., "Alternating versus hybrid MOPP and ABVD combinations in advanced Hodgkin's disease: ten-year results," Journal of Clinical Oncology, vol. 14, no. 5, pp. 1421-1430, 1996.

[41] J. M. Connors, P. Klimo, G. Adams et al., "Treatment of advanced Hodgkin's disease with chemotherapy_comparison of MOPP/ABV hybrid regimen with alternating courses of MOPP and ABVD: a report from the National Cancer Institute of Canada Clinical Trials Group," Journal of Clinical Oncology, vol. 15, no. 4, pp. 1638-1645, 1997.

[42] D. B. Duggan, G. Petroni, J. Johnson et al., "MOPP/ABV versus ABVB for advanced Hodgkin's disease-preliminary report of CALGB 8952," Proceedings of the American Society of Clinical Oncology, vol. 16, p. 12, 1997, abstract 43.

[43] A. Engert and M. Dreyling, "Hodgkin's lymphoma: ESMO clinical recommendations for Diagnosis, treatment and follow-up," Annals of Oncology, vol. 19, supplement 2, pp. 6566, 2008.

[44] E. Brusamolino, A. Bacigalupo, G. Barosi et al., "Classical Hodgkin's lymphoma in adults: guidelines of the Italian Society of hematology, the Italian Society of Experimental hematology, and the Italian Group for Bone Marrow Transplantation on initial work-up, management, and follow-up," Haematologica, vol. 94, no. 4, pp. 550-565, 2009.

[45] M. Hutchings, N. G. Mikhaeel, P. A. Fields, T. Nunan, and A. R. Timothy, "Prognostic value of interim FDG-PET after two or three cycles of chemotherapy in Hodgkin lymphoma," Annals of Oncology, vol. 16, no. 7, pp. 1160-1168, 2005.

[46] N. Tsukamoto, M. Kojima, M. Hasegawa et al., "The usefulness of 18F-fluorodeoxyglucose positron emission tomography (18F-FDG-PET) and a comparison of 18F-FDG-PET with 67 gallium scintigraphy in the evaluation of lymphoma: relation to histologic subtypes based on the World Health Organization classification," Cancer, vol. 110, no. 3, pp. 652659, 2007.

[47] T. Girinsky, M. Ghalibafian, G. Bonniaud et al., "Is FDGPET scan in patients with early stage Hodgkin lymphoma of any value in the implementation of the involved-node radiotherapy concept and dose painting?" Radiotherapy and Oncology, vol. 85, no. 2, pp. 178-186, 2007. 
[48] G. P. Canellos, "Chemotherapy alone for early Hodgkin's lymphoma: an emerging option," Journal of Clinical Oncology, vol. 23, no. 21, pp. 4574-4576, 2005.

[49] R. M. Meyer, "Is there convincing evidence for the use of chemotherapy alone in patients with limited stage Hodgkin's lymphoma?" European Journal of Haematology, vol. 75, no. 66, supplement, pp. 115-120, 2005.

[50] G. P. Canellos, J. S. Abramson, D. C. Fisher, and A. S. LaCasce, "Treatment of favorable, limited-stage Hodgkin's lymphoma with chemotherapy without consolidation by radiation therapy," Journal of Clinical Oncology, vol. 28, no. 9, pp. 1611-1615, 2010.

[51] "NCCN Practice Guidelines in Oncology: Hodgkin's Disease/ Lymphoma," vol. 2, 2008.

[52] C. Herbst, F. A. Rehan, C. Brillant et al., "Combined modality treatment improves tumor control and overall survival in patients with early stage Hodgkin's lymphoma: a systematic review," Haematologica, vol. 95, no. 3, pp. 494-500, 2010.

[53] L. Nogová, T. Reineke, C. Brillant et al., "Lymphocytepredominant and classical Hodgkin's lymphoma: a comprehensive analysis from the german Hodgkin study group," Journal of Clinical Oncology, vol. 26, no. 3, pp. 434-439, 2008.

[54] L. Nogová, T. Reineke, H. T. Eich et al., "Extended field radiotherapy, combined modality treatment or involved field radiotherapy for patients with stage IA lymphocytepredominant Hodgkin's lymphoma: a retrospective analysis from the German Hodgkin Study Group (GHSG)," Annals of Oncology, vol. 16, no. 10, pp. 1683-1687, 2005.

[55] D. L. Longo, R. C. Young, and M. Wesley, "Twenty years of MOPP therapy for Hodgkin's disease," Journal of Clinical Oncology, vol. 4, no. 9, pp. 1295-1306, 1986.

[56] D. B. Duggan, G. R. Petroni, J. L. Johnson et al., "Randomized comparison of ABVD and MOPP/ABV hybrid for the treatment of advanced Hodgkin's disease: report of an Intergroup trial," Journal of Clinical Oncology, vol. 21, no. 4, pp. 607-614, 2003.

[57] A. Santoro, G. Bonadonna, and P. Valagussa, "Long-term results of combined chemotherapy-radiotherapy approach in Hodgkin's disease: superiority of ABVD plus radiotherapy versus MOPP plus radiotherapy," Journal of Clinical Oncology, vol. 5, no. 1, pp. 27-37, 1987.

[58] P. W. M. Johnson, J. A. Radford, M. H. Cullen et al., "Comparison of ABVD and alternating or hybrid multidrug regimens for the treatment of advanced Hodgkin's lymphoma: results of the United Kingdom Lymphoma Group LY09 Trial," Journal of Clinical Oncology, vol. 23, no. 36, pp. 9208-9218, 2005.

[59] V. Diehl, J. Franklin, M. Pfreundschuh et al., "Standard and increased-dose BEACOPP chemotherapy compared with COPP-ABVD for advanced Hodgkin's disease," New England Journal of Medicine, vol. 348, no. 24, pp. 2386-2395, 2003.

[60] S. J. Horning, R. T. Hoppe, S. Breslin, N. L. Bartlett, B. William Brown, and S. A. Rosenberg, "Stanford V and radiotherapy for locally extensive and advanced Hodgkin's disease: mature results of a prospective clinical trial," Journal of Clinical Oncology, vol. 20, no. 3, pp. 630-637, 2002.

[61] P. G. Gobbi, A. Levis, T. Chisesi et al., "ABVD versus modified Stanford V versus MOPPEBVCAD with optional and limited radiotherapy in intermediate- and advanced-stage Hodgkin's lymphoma: final results of a multicenter randomized trial by the Intergruppo Italiano Linfomi," Journal of Clinical Oncology, vol. 23, no. 36, pp. 9198-9207, 2005.

[62] M. Loeffler, O. Brosteanu, D. Hasenclever et al., "Meta-analysis of chemotherapy versus combined modality treatment trials in
Hodgkin's disease," Journal of Clinical Oncology, vol. 16, no. 3, pp. 818-829, 1998.

[63] C. Fermé, C. Sebban, C. Hennequin et al., "Comparison of chemotherapy to radiotherapy as consolidation of complete or good partial response after six cycles of chemotherapy for patients with advanced Hodgkin's disease: results of the Groupe d'etudes des Lymphomes de l'Adulte H89 trial," Blood, vol. 95, no. 7, pp. 2246-2252, 2000.

[64] B. M. P. Aleman, J. M. M. Raemaekers, U. Tirelli et al., "Involved-field radiotherapy for advanced Hodgkin's lymphoma," New England Journal of Medicine, vol. 348, no. 24, pp. 2396-2406, 2003.

[65] P. W. Johnson, M. R. Sydes, B. W. Hancock, M. Cullen, J. A. Radford, and S. P. Stenning, "Consolidation radiotherapy in patients with advanced Hodgkin's lymphoma: survival data from the UKLG LY09 randomized controlled trial (ISRCTN97144519)," Journal of Clinical Oncology, vol. 28, no. 20, pp. 3352-3359, 2010.

[66] C. Kobe, M. Dietlein, J. Franklin et al., "Positron emission tomography has a high negative predictive value for progression or early relapse for patients with residual disease after first-line chemotherapy in advanced-stage Hodgkin lymphoma," Blood, vol. 112, no. 10, pp. 3989-3994, 2008.

[67] J. C. Poen, R. T. Hoppe, and S. J. Horning, "High-dose therapy and autologous bone marrow transplantation for relapsed/refractory Hodgkin's disease: the impact of involved field radiotherapy on patterns of failure and survival," International Journal of Radiation Oncology Biology Physics, vol. 36, no. 1, pp. 3-12, 1996.

[68] C. H. Moskowitz, S. D. Nimer, A. D. Zelenetz et al., "A 2-step comprehensive high-dose chemoradiotherapy secondline program for relapsed and refractory Hodgkin disease: analysis by intent to treat and development of a prognostic model," Blood, vol. 97, no. 3, pp. 616-623, 2001.

[69] J. Yahalom, S. C. Gulati, M. Toia et al., "Accelerated hyperfractionated total-lymphoid irradiation, high-dose chemotherapy, and autologous bone marrow transplantation for refractory and relapsing patients with Hodgkin's disease," Journal of Clinical Oncology, vol. 11, no. 6, pp. 1062-1070, 1993. 


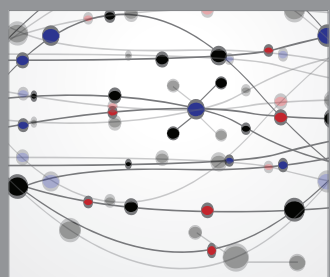

The Scientific World Journal
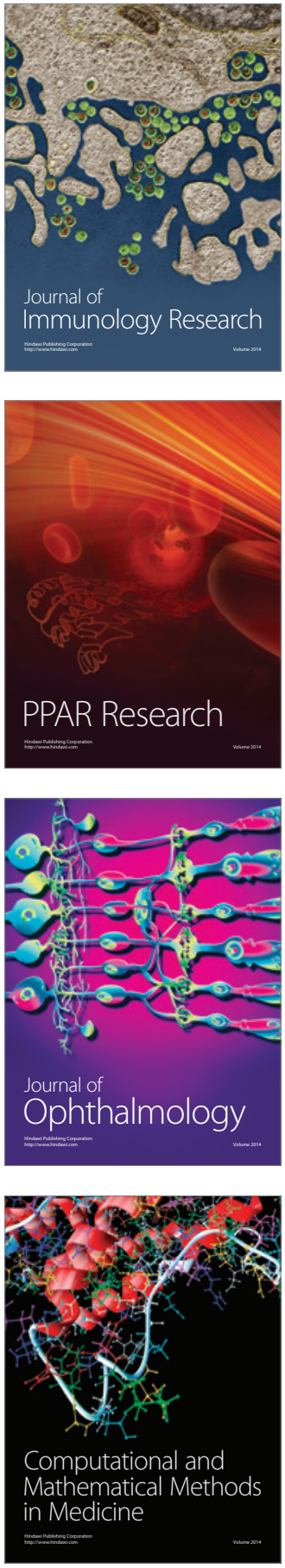

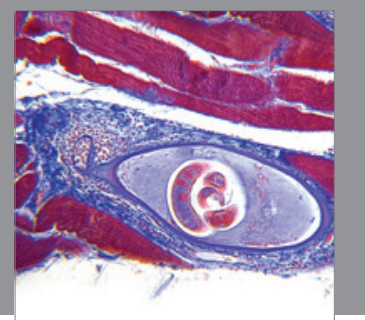

Gastroenterology

Research and Practice
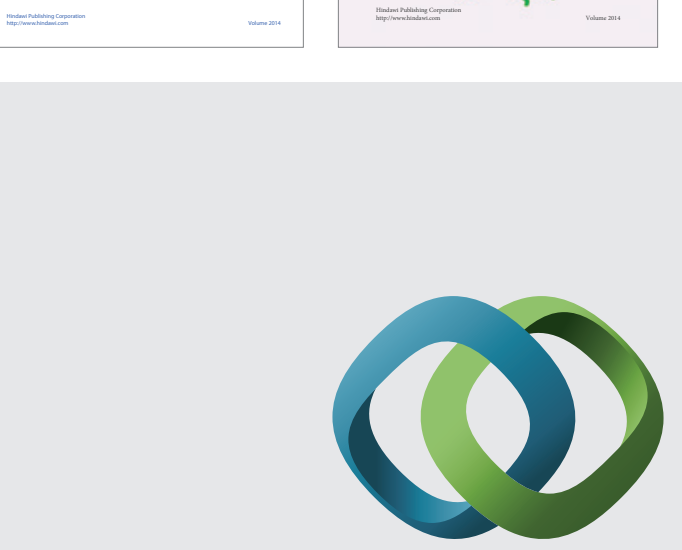

\section{Hindawi}

Submit your manuscripts at

http://www.hindawi.com
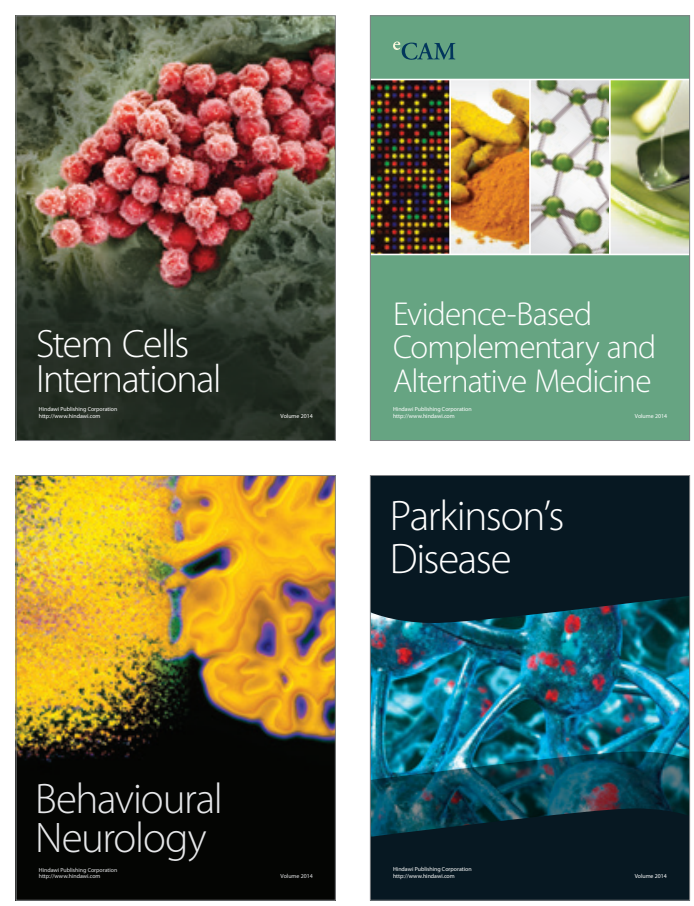

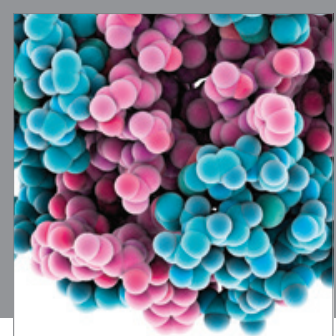

Journal of
Diabetes Research

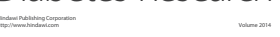

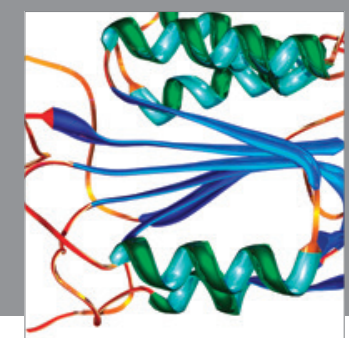

Disease Markers
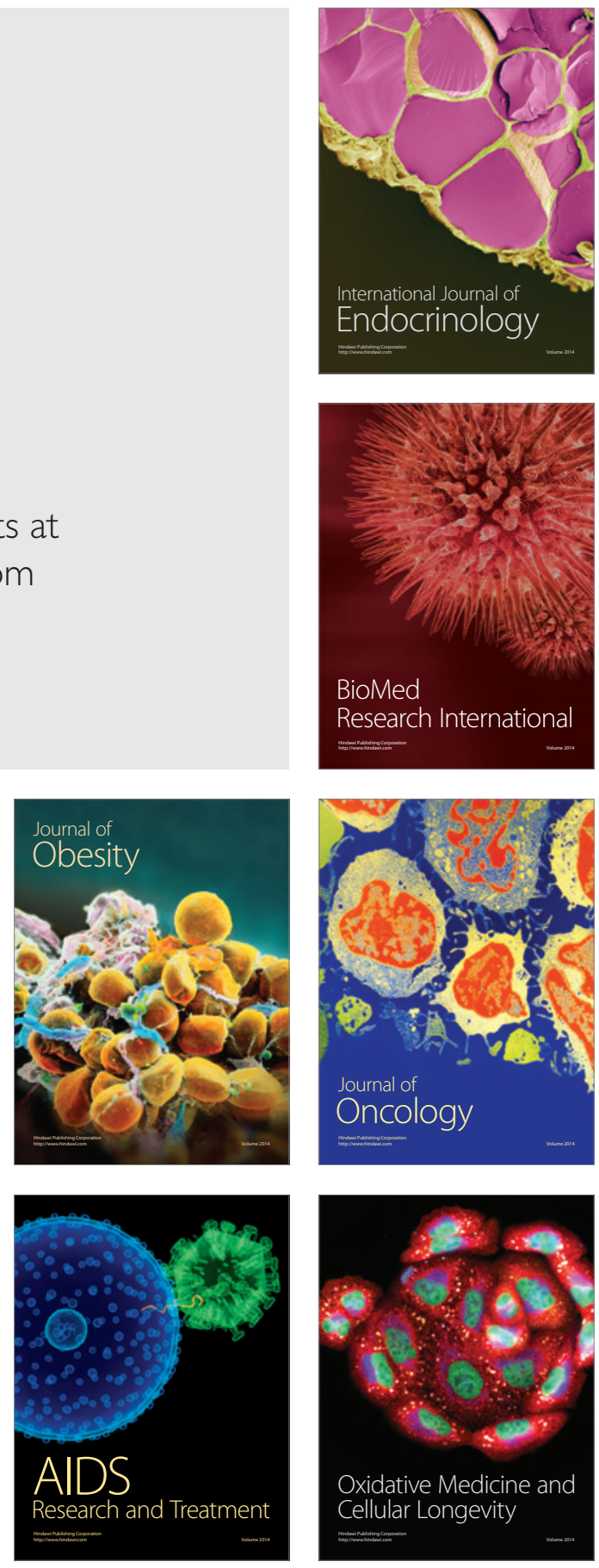